\title{
Bifurcations Are Not Always Exclusive
}

\author{
SARAH SMITHERMAN PRATT \\ The University of North Carolina at Greensboro (USA)
}

Non-linear dynamics emerged in the fields of science and mathematics at the turn of the

$20^{\text {th }}$ Century. Since that time, many researchers employ ideas associated with non-linear patterns of interactions. In science, complexity theorists study emergence, feedback loops, and self-organization in systems. In mathematics, chaos theorists examine patterns that functions display when iterated. (Iteration is the process of using the output as a new input.) Essentially, complexity theory is the study of order emerging from disorder, while chaos theory is the study of disorder emerging out of order (specifically how a controlled environment can behave unpredictably). Ironically, though, as each group seeks to explore and explain emergent patterns in systems and label a pattern as a "bifurcation," complexity theorists discern and exclude, while chaos theorists include and embrace simultaneities.

The bifurcation diagram (see Figure 1), which is often referenced in non-linear dynamics, is similar to the Mandelbrot Set. The Mandelbrot Set depicts the set of all Julia sets; the bifurcation diagram is the set of all iterated functions (varying of initial seeds) for different constant or "control" parameter values. The bifurcation diagram, at first glance appears to be an imagined series of "forks in the road." One reason for this misinterpretation is the misreading of the independent variable (horizontal axis) as time and the dependent variable (vertical axis) as a result of the function with respect to time. What the graph actually depicts, however, is what patterns emerge when a function is iterated as its control parameter is varied. 


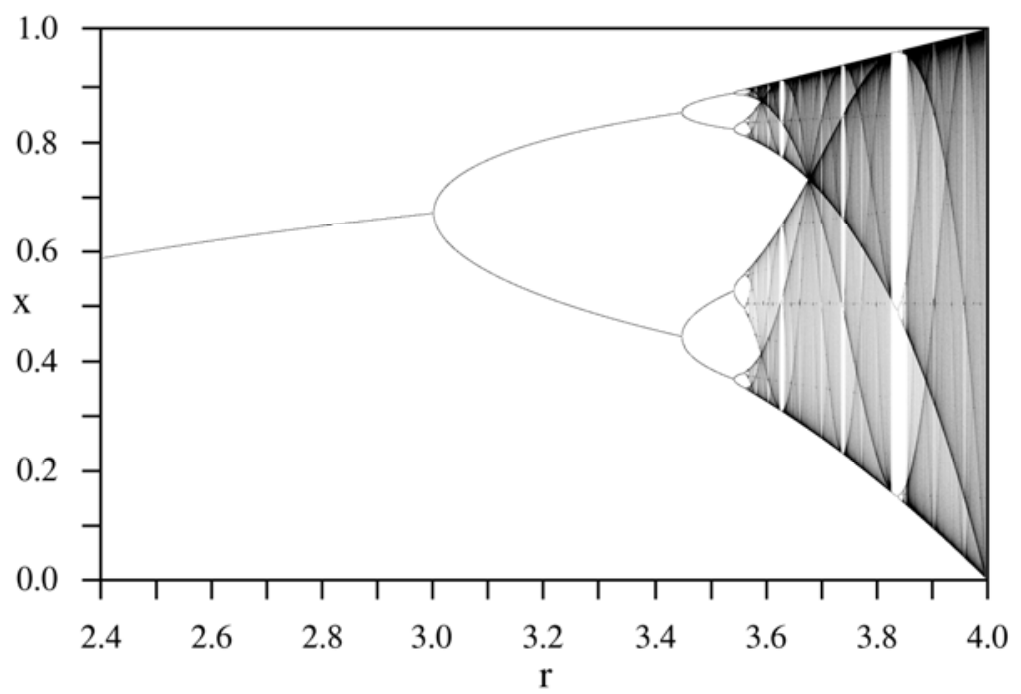

Figure 1. Bifurcation diagram of the Logistic map (Wikipedia Commons, 2005).

A second reason for this misinterpretation is due to the disparate definitions of the word "bifurcation" as it is currently used in non-linear dynamics. In complexity theory the interpretation is based on Ilya Prigogine's (1977) pioneering work of dissipative structures, states of equilibrium, and self-organization. Prigogine describes, in his Nobel laureate lecture, the concept of successive bifurcations and how the increase of the control parameter leads to moments of bifurcations in which the system self-organizes into different states, or paths (p. 273). Other complexity theorists assume this perspective of bifurcations, relating to "choices" or "decisions," and describe intended movements toward one path of self-organization or another, as well as analyzing systems and their states of equilibrium (e.g., Taylor, 2001, p. 150). On/Off, binary oppositions, 0/1 are all examples of mutually exclusive bifurcations. In traditional logic, exclusive "or" means that one condition must be true but the other must not in order for the statement to be true. Inclusive "or" allows a statement to be true if one or the other condition is true, and both conditions can be true. Bifurcations in chaos theory mathematics maintain the logic of inclusive "or" in which a function reveals moments of one value then moves to another value. These values are part of the pattern, part of the system. Together they describe characteristics about the system. The differences are not separate distinctions; rather, the differences enhance the qualities of the system.

Neither Prigogine's (2000) definition of "successions of bifurcations, [which introduces] an 'historical' element" (p. 894) nor his successive bifurcations diagram (1977, p. 273) mean the same as the bifurcation diagram used in chaos theory of mathematics to display patterns of iterated functions. The two diagrams are not interchangeable. The former relies on exclusive "or," while the latter is dependent upon inclusive "or."

The bifurcation, or orbit, diagram explores the patterns of possibilities. (Orbit diagram may be a better descriptor than bifurcation diagram to separate what the graph displays.) An orbit analysis-a demonstration of what values the function oscillates 
between or among - reveals what patterns may emerge when the function is repeatedly iterated. One particular function often examined in chaos theory mathematics is the logistic function, which can be expressed as the iterative function: $x_{\mathrm{k}+1}=\lambda x_{\mathrm{k}}\left(1-x_{\mathrm{k}}\right)$, or generally as $\mathrm{F}(x)=\lambda x(1-x)$ (e.g., Devaney, 1992, p. 12). For every $\lambda$ that is selected, the function is iterated with varying initial seeds $\left(x_{\mathrm{k}}\right)$. This function is of particular interest because there are points at which the function displays orbits of 2-cycle, 4-cycle, 8-cycle, and between the control parameters values of 3.5 and 4.0, chaotic patterns occur.

Robert Devaney's (n.d.) web site contains an orbit diagram of the logistic map using a Java Applet (created by Yakov Shapiro). This diagram dynamically illustrates how a logistic function moves to a particular orbit based on the initial seed of $x=0.5$. The first 100 iterations are omitted from the graph to show how the function eventually "settles down." For example, entering $C=3.2$ and clicking "display orbit" reveals the 2-cycle orbit values that occur (highlighted in red), and the column to the right displays the values of the iterations. Entering $C=3.5$ and clicking "display orbit" shows the 4-cycle orbit values that result. Entering $C=3.7$ and clicking "display orbit" shows the chaotic pattern, with no specific orbit, that emerges from the iterations. Time is not part of either axis of the graph. The orbit diagram depicts what happens to the iterated values for different control parameters. With these analyses chaos theorists are attempting to control and predict, even when predicting includes unpredictability.

From my perspective, I employ a postmodern logic of interpretation associated with hermeneutics, a perspective that dismisses control because it is merely an illusion. I strive in my own teaching to have shifts in dialogues in non-linear ways during class time (Smitherman, 2005, p. 161). Instead of looking from left to right as time passes, I try to shift the initial seed to something generative that creates complex conversations (Pratt, 2008). Non-linear interpretations of discourse include patterns of emergence and of interactions within a system, not a mechanistic, cause-effect interpretation of requiring this to be implemented in order for that to happen. Education perceived from a postmodern logic and a systems theory perspective embraces interactions and moments of difference rather than patterns of control that render conformity.

\section{References}

Devaney, R. (n.d.) Orbit diagram applets. Bob Devaney's home page. Boston, MA: Boston University. Retrieved on-line on May 23, 2008, at http://math.bu.edu/DYSYS/applets/bif-dgm/Logistic.html.

Devaney, R. (1992). A first course in chaotic dynamical systems: Theory and experiment. Cambridge, MA: Perseus Books.

Pratt, S. (2008). Complexity, constructivism, and the power dynamics of "understanding." Journal of Canadian Association for Curriculum Studies. 6(1) 113-132. Retrieved 22 July 2008 at http://www.csse.ca/CACS/JCACS/V6N1/PDFContent/05\%20Sarah\%20Pratt\%20FINAL.pdf

Prigogine, I. (2000). The networked society. Journal of World-Systems Research, VI (3), 892-898.

Prigogine, I. (1977, December). Time, structure and fluctuations. Nobel Lecture. Université Libre de Bruxelles, Brussels, Belgium. Retrieved online on May 25, 2008, from http://nobelprize.org/nobel prizes/chemistry/laureates/1977/prigogine-lecture.pdf.

Smitherman, S. (2005). Creating holes and wholes in curriculum. In W. Doll, J. Fleener, D. Trueit, \& J., St. Julien (Eds.). Chaos, Complexity, Curriculum and Culture: A Conversation (pp. 153-180). New York: Peter Lang. 
Taylor, M. (2001). The moment of complexity: Emerging network culture. Chicago, IL: University of Chicago Press.

Wikipedia Commons. (2005). Bifurcation diagram of the Logistic map for parameter r=2.4 to 4.0. Wikipedia. San Francisco, CA: Wikimedia Foundation, Inc. Retrieved online on June 9, 2008, from http://en.wikipedia.org/wiki/Image:LogisticMap BifurcationDiagram.png [public domain].

\begin{abstract}
About the Author
Sarah Smitherman Pratt is an assistant professor in the Department of Curriculum and Instruction at the University of North Carolina at Greensboro, where she focuses on middle grades and secondary mathematics education. She is currently researching complex conversations and how these types of interactions can promote mathematical thinking in the classroom. [Email: sspratt@uncg.edu; web site (currently under construction): http://www.uncg.edu/cui/sarah pratt/index.html.]
\end{abstract}

(C) Copyright 2008. The author, SARAH SMITHERMAN PRATT, assigns to the University of Alberta and other educational and non-profit institutions a non-exclusive license to use this document for personal use and in courses of instruction provided that the article is used in full and this copyright statement is reproduced. The author also grants a non-exclusive license to the University of Alberta to publish this document in full on the World Wide Web, and for the document to be published on mirrors on the World Wide Web. Any other usage is prohibited without the express permission of the author. 